\title{
An Asymptotic Expansion for the Multivariate Normal Distribution and Mills' Ratio
}

\author{
Harold Ruben*
}

(September 20, 1963)

\begin{abstract}
An asymptotic expansion for the multivariate normal integral over an infinitely extended rectangle, and therefore also for the associated multivariate Mills' ratio, is developed. The expansion is valid provided the vertex of the rectangle lies in a polyhedral half-cone determined by the set of regression planes.

The expansion obtained here is a natural generalization of the classic expansion for the normal univariate integral, and the coefficients in it involve the moments of the conjugate multinormal distribution.
\end{abstract}

\section{Introduction}

Define

and

$$
\begin{aligned}
f(\mathbf{x}, \mathbf{M}) & =(2 \pi)^{-n / 2}|\mathbf{M}|^{1 / 2} e^{-\mathbf{x} \mathbf{M} \mathbf{x}^{\prime} / 2} \\
F(\mathbf{a}, \mathbf{M}) & =\int_{\mathbf{x} \geqq \mathbf{a}} f(\mathbf{x}, \mathbf{M}) d \mathbf{x}
\end{aligned}
$$

$$
R(\mathbf{a}, \mathbf{M})=F(\mathbf{a}, \mathbf{M}) / f(\mathbf{a}, \mathbf{M}) .
$$

Thus, $f(\mathbf{x}, \mathbf{M})$ is the probability density of a random normal vector $\mathbf{X}=\left(X_{1}, \ldots, X_{n}\right)$ with expectation vector zero and nonsingular variance-covariance matrix $\mathbf{M}^{-1} ; F(\mathbf{a}, \mathbf{M})$ is the probability that $\mathbf{X} \geqq \mathbf{a}$ for a fixed vector $\mathbf{a}=\left(a_{1}, \ldots, a_{n}\right)$, where $\mathbf{X} \geqq \mathbf{a}$ is to be interpreted as the set of simultaneous inequalities $X_{\alpha} \geqq a_{\alpha}(\alpha=1, \ldots, n)$; finally, $R(\mathbf{a}, \mathbf{M})$ is (the $n$-variate) Mills' ratio for a multinormal distribution in the sense that it represents the ratio of the probability-mass in the infinitely extended $n$-dimensional rectangle $\mathbf{x} \geqq \mathbf{a}$ to the probability density at the vertex, a, of the rectangle. (This point is frequently referred to in this context as the "cutoff point.") Indeed, for the special case $n=1$, with $\mathbf{M}=1$, we have the usual (univariate) Mills' ratio

$$
R(a, 1)=\int_{a}^{\infty}(2 \pi)^{-1 / 2} e^{-x^{2} / 2} d x /\left\{(2 \pi)^{-1 / 2} e^{-a^{2} / 2}\right\} .
$$

In a recent paper in this journal, I. R. Savage ${ }^{1}[14]^{2}$ has obtained two useful and easily applied inequalities for $R(\mathbf{a}, \mathbf{M})$, and therefore also for $F(\mathbf{a}, \mathbf{M})$, when $\mathbf{a M}>\mathbf{0}$. We shall here obtain an asymptotic expansion for $R(\mathbf{a}, \mathbf{M})$ under the same conditions which produces a sequence of upper and lower bounds for $R(\mathbf{a}, \mathbf{M})$ and $F(\mathbf{a}, \mathbf{M})$. Savage's inequalities correspond to the first of these upper and lower bounds.

\footnotetext{
*Department of Statistics, University of Minnesota.

'The notation adopted in this paper is that of Savage. It should be remarked that Savage's results and the results of the present paper were obtained inde-

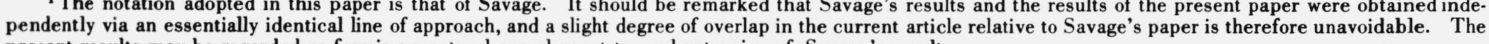
present results may be regarded as forming a natural complement to, and extension of, Savage's results.

${ }^{2}$ Figures in brackets indicate the literature references at the end of this paper.
} 


\section{Derivation of the Expansion and Discussion of Its Properties}

On setting

$$
\mathbf{y}=\mathbf{x}-\mathbf{a}
$$

(1.2) becomes

$$
F(\mathbf{a}, \mathbf{M})=f(\mathbf{a}, \mathbf{M}) \cdot \int_{\mathbf{y} \geqq 0} e^{-\Delta \mathbf{y}^{\prime}-\mathbf{y M} \mathbf{y}^{\prime} / 2} d \mathbf{y},
$$

where

$$
\Delta=\mathbf{a M}
$$

that is,

$$
R(\mathbf{a}, \mathbf{M})=\int_{\mathbf{y} \geqq 0} e^{-\Delta \mathbf{y}^{\prime}-\mathbf{y M} \mathbf{y}^{\prime} / 2} d \mathbf{y} .
$$

To obtain a series expansion for $R(\mathbf{a}, \mathbf{M})$ from (2.3), expand $\exp \left(-\mathbf{y} \mathbf{M} \mathbf{y}^{\prime} / 2\right)$ in its power series form round $\mathbf{y}=\mathbf{0}$ and, on the assumption that $\Delta>\mathbf{0}$, integrate term by term. (The sense in which this purely formal procedure is justified is described below.) We note that $\mathbf{y} \mathbf{M} \mathbf{y}^{\prime}$ is symmetric round $\mathbf{y}=\mathbf{0}$, remaining unaffected when $\mathbf{y}$ is replaced by $-\mathbf{y}$, so that

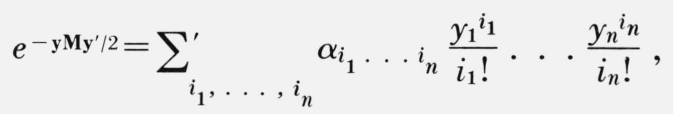

where

$$
\alpha_{i_{1}} \ldots i_{n}=\left.\left(\partial^{i_{1}+\ldots+i_{n}} / \partial y_{1}{ }^{i_{1}} \ldots \partial y_{n}{ }^{i} n\right) e^{-\mathbf{y M} \mathbf{y}^{\prime} / 2}\right|_{\mathbf{y}=\mathbf{0}}
$$

and $\Sigma^{\prime}{ }_{i}, \ldots, i_{n}$ denotes summation over all nonnegative integral $i_{1}, \ldots, i_{n}$ such that $i_{1}+\ldots+i_{n}$ is an even integer (including zero). Consider now a random normal vector $\mathbf{X}^{*}=\left(X_{1}^{*}, \ldots, X_{n}^{*}\right)$ with expectation vector zero and variance-covariance matrix $\mathbf{M}$ : such a vector may be described as having a distribution conjugate to that of $\mathbf{X}$. The coefficients $\alpha_{i_{1}} \ldots i_{n}$ in the above series representation of $\exp \left(-\mathbf{y} \mathbf{M} \mathbf{y}^{\prime} / 2\right)$ can then be expressed in terms of the moments of the conjugate distribution. For since the characteristic function of $\mathbf{X}^{*}$ is given by

$$
E\left[e^{i \mathrm{X} \mathbf{X}^{* \prime}}\right]=e^{-\mathbf{t M t} / 2},
$$

t denoting a fixed $n$-dimensional vector with real components, we have

$$
\alpha_{i_{1}} \ldots i_{n}=i^{i_{1}+\ldots+i_{n}} \mu_{i_{1}}^{*} \ldots i_{n},
$$

where

$$
\mu_{i_{1}}^{*} \ldots i_{n}=E\left[\left(X_{1}^{*}\right)^{i_{1}} \ldots\left(X_{n}^{*}\right)^{i_{n}}\right]
$$

Hence (2.3) can be expressed in the form

$$
R(\mathbf{a}, \mathbf{M})=\int_{0}^{\infty} \ldots \int_{0}^{\infty} e_{\alpha=1}^{-\sum_{\alpha}} \Delta_{\alpha} y_{\alpha} \sum_{i_{1}, \ldots, i_{n}}^{\prime}(-1)^{\left(i_{1}+\ldots+i_{n}\right) / 2} \mu_{i_{1}}^{*} \ldots i_{n} \frac{y_{1}^{i_{1}}}{i_{1} !} \ldots \frac{y_{n}^{i_{n}}}{i_{n} !} d y_{1} \ldots d y_{n},
$$

and subsequent term by term integration yields the result that $R(\mathbf{a}, \mathbf{M})$ can be formally identified 
with the $n$-variate series

$$
\begin{aligned}
& \sum_{i_{1}, \ldots, i_{n}}^{\prime}(-1)^{\left(i_{1}+\ldots+i_{n}\right) / 2} \mu_{i_{1}}^{*} \ldots i_{n} \prod_{\alpha=1}^{n} \int_{0}^{\infty} e^{-\Delta_{\alpha} y_{\alpha}} \frac{y_{\alpha}{ }^{i_{\alpha}}}{i_{\alpha} !} d y_{i_{\alpha}} \\
& \quad=\sum_{i_{1}, \ldots, i_{n}}^{\prime}(-1)^{\left(i_{1}+\ldots+i_{n}\right) / 2} \mu_{i_{1}}^{*} \ldots i_{n} \prod_{\alpha=1}^{n} \frac{1}{\Delta_{\alpha}{ }^{i} \alpha^{+1}} \quad\left(\Delta_{\alpha}>0 ; \alpha=1, \ldots, n\right) .
\end{aligned}
$$

Laplace's classic asymptotic expansion, $a$ large, for the univariate Mills' ratio, as defined in (1.4), is

$$
R(a, 1) \sim \frac{1}{a} \cdot\left\{1-\frac{1}{a^{2}}+\frac{1.3}{a^{4}}-\frac{1.3 .5}{a^{6}}+\ldots\right\} \quad(a>0)
$$

the symbol $\sim$ denoting the fact that the series on its right is an asymptotic representation of the function on its left. If $X$ is a (univariate) normal random variable with zero mean and unit standard deviation, then $X^{*}$, the conjugate random variable, has the same distribution. The coefficient of $1 / a^{2 j}$ in the bracketed expression in $(2.6)$ is $(-1)^{j} 1.3 .5 \ldots(2 j-1)$, and this is the $(2 j)$ th moment of $X^{*}$ about the origin. From this point of view it is clear that (2.5) represents the multivariate analog of (2.6), since the $\mu_{i_{1}}^{*} \ldots i_{n}$ are the moments about the origin of the distribution of $\mathbf{X}^{*}$, i.e., of the distribution conjugate to that of $\mathbf{X}$. However, the analogy is exhibited most clearly if (2.5) is expressed in more explicit form as

$$
R(\mathbf{a}, \mathbf{M}) \sim \frac{1}{\Delta_{1} \ldots \Delta_{n}} \cdot\left\{C_{0}-C_{1}+C_{2}-\ldots\right\} \quad\left(\Delta_{\alpha}>0 ; \alpha=1, \ldots, n\right),
$$

where

$$
C_{j}=\sum_{i_{1}+\ldots+i_{n}=2 j} \frac{\mu_{i_{1}}^{*} \ldots i_{n}}{\Delta_{1_{1}}^{i_{1}} \ldots \Delta_{n^{n}}^{i_{n}}} \quad(j=0,1, \ldots) .
$$

(For a proof that the symbol $\sim$ is justified in (A), see below.) Expansion (A) is also the form to be used for computing purposes.

The first three $C_{j}$ are given by

$C_{0}=1$,

$$
\begin{aligned}
& C_{1}=\left(\frac{\mu_{20}^{*} \ldots 0}{\Delta_{1}^{2}}+\ldots+\frac{\mu_{0}^{*} \ldots 02}{\Delta_{n}^{2}}\right)+\left(\frac{\mu_{110}^{*} \ldots 0}{\Delta_{1} \Delta_{2}}+\frac{\mu_{1010}^{*} \ldots 0}{\Delta_{1} \Delta_{3}}+\ldots+\frac{\mu_{0}^{*} \ldots 011}{\Delta_{n-1} \Delta_{n}}\right), \\
& C_{2}=\left(\frac{\left.\mu_{40 \ldots 0}^{*} \ldots . .+\frac{\mu_{0}^{*} \ldots 04}{\Delta_{1}^{4}}+\ldots\right)}{\Delta_{n}^{4}}\right) \\
& +\left(\frac{\mu_{310 \ldots 0}^{*} \ldots 0}{\Delta_{1}^{3} \Delta_{2}}+\frac{\mu_{3010 \ldots 0}^{*} \ldots}{\Delta_{1}^{3} \Delta_{3}}+\ldots+\frac{\mu_{0}^{*} \ldots 031}{\Delta_{n-1}^{3} \Delta_{n}}+\frac{\mu_{130 \ldots 0}^{*} \ldots 0}{\Delta_{1} \Delta_{2}^{3}}+\frac{\mu_{1030 \ldots 0}^{*} \ldots 0}{\Delta_{1} \Delta_{3}^{3}}+\frac{\mu_{0}^{*} \ldots 013}{\Delta_{n-1} \Delta_{n}^{3}}\right) \\
& +\left(\frac{\mu_{220}^{*} \ldots 0}{\Delta_{1}^{2} \Delta_{2}^{2}}+\frac{\mu_{2020 \ldots 0}^{*} \ldots}{\Delta_{1}^{2} \Delta_{3}^{2}}+\ldots+\frac{\mu_{0}^{*} \ldots 022}{\Delta_{n-1}^{2} \Delta_{n}^{2}}\right) \\
& +\left(\frac{\mu_{2110 \ldots 0}^{*} \ldots .}{\Delta_{1}^{2} \Delta_{2} \Delta_{3}}+\ldots\right)+\left(\frac{\mu_{1110}^{*} \ldots 0}{\Delta_{1} \Delta_{2} \Delta_{3} \Delta_{4}}+\ldots\right),
\end{aligned}
$$

the different bracketed terms in $C_{1}$ and $C_{2}$ corresponding to the various partitionings of 2 and 4 , respectively, into $n$ nonnegative integers. (The last two bracketed terms in (2.9) vanish if $n=2$, 
and the last bracketed term vanishes if $n=3$.) More generally, it is clear from (A.1) that the evaluation of $C_{j}$ requires the enumeration of all possible partitionings of $2 j$ into $n$ nonnegative integers.

The expansion (A) for the multivariate Mills' ratio has some useful properties which are quite analogous to those of the corresponding expansion (2.6) for the univariate Mills' ratio. The properties in question are as follows:

(i) Expansion (A) is an enveloping one, in that $R(\mathbf{a}, \mathbf{M})$ falls short of every summand with an odd number of terms and exceeds every summand with an even number of terms.

(ii) The truncation error after any number of terms in (A) is numerically less than the firsi term omitted.

(iii) Expansion (A) is asymptotic for large $\Delta_{\alpha}$ (as remarked previously after (A.1)).

We now proceed to establish these properties. First, recall that (2.5) was obtained by expanding $\exp \left(-\mathbf{y} \mathbf{M} \mathbf{y}^{\prime} / 2\right)$ as a power series in the $y_{\alpha}$ and integrating term by term in (2.3). Correspondingly, (A) can be obtained by expanding $\exp \left(-\mathbf{y} \mathbf{M} \mathbf{y}^{\prime} / 2\right)$ as a power series in $\mathbf{y} \mathbf{M} \mathbf{y}^{\prime}$ and integrating term by term in (2.3); that is,

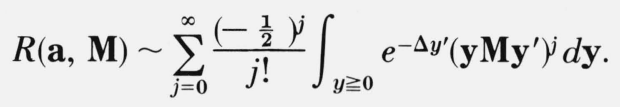

Therefore, on using the generalized mean-value theorem, the error after $m$ terms, $E_{m}$, is given by

$$
E_{m}=\frac{\left(-\frac{1}{2}\right)^{m}}{m !} \int_{\mathbf{y} \geqq 0} e^{-\Delta \mathbf{y}^{\prime}}\left(\mathbf{y} \mathbf{M} \mathbf{y}^{\prime}\right)^{m} e^{-\zeta \mathbf{M} \zeta^{\prime} / 2} d \mathbf{y}\left(\zeta \mathbf{M} \zeta^{\prime}<\mathbf{y} \mathbf{M} \mathbf{y}^{\prime}\right)
$$

Hence $E_{m}$ is positive if $m$ is even and negative if $m$ is odd. This proves the enveloping property.

Next from (2.11).

$$
\left|E_{m}\right|<\frac{\left(\frac{1}{2}\right)^{m}}{\mathrm{~m} !} \int_{\mathbf{y} \geqq \mathbf{0}} e^{-\Delta \mathbf{y}^{\prime}}\left(\mathbf{y} \mathbf{M} \mathbf{y}^{\prime}\right)^{m} d \mathbf{y}
$$

and since the right-hand member in the inequality $(2.12)$ is numerically equal to the $(m+1)$ th term in the expansion (2.10), which is also the expansion (A), the second of the above-mentioned three properties is proved.

Finally, to prove the asymptotic nature of (A), observe that in conjunction with the result just proved about the magnitude of the truncation error the desired result is achieved if it can be shown that the successive terms in (A) are in decreasing order of magnitude. It is clear from direct inspection that the latter terms do, in fact, stand in this relation to one another for sufficiently large $\Delta_{\alpha}(\alpha=1, \ldots, n)$, and correspondingly that the series in (A), and its equivalent (2.10), do represent asymptotic representations of $R(\mathbf{a}, \mathbf{M})$ for sufficiently large $\Delta_{1}, \ldots, \Delta_{n}$ (use of the symbol $\sim$ in the two equivalent formulas (A) and (2.10) being thereby justified). We shall, however, strengthen this by demonstrating that (A) is an asymptdtic representation of $R(\mathbf{a}, \mathbf{M})$ for sufficiently large values of $\Sigma_{1}^{n} \Delta_{\alpha}{ }^{2}$. This means that (A) can be used profitably even if some of the individual $\Delta_{\alpha}$ are small or moderately large, provided only that $\Sigma_{1}^{n} \Delta_{\alpha}^{2}$ is large. Indeed, on setting

$$
\begin{aligned}
& \mathbf{y}=r \mathbf{l} \quad(|\mathbf{l}|=1), \\
& L=\Delta \mathbf{l}^{\prime}, Q=\mathbf{l M l}^{\prime}
\end{aligned}
$$

(l is a unit $n$-dimensional vector parallel to $\mathbf{y}$ ), the series in (2.10) transforms to

$$
\sum_{j=0}^{\infty} \frac{\left(-\frac{1}{2}\right)^{j}}{j !} \int_{0}^{\infty} \int_{O_{n}} e^{-L r} Q^{j} r^{2 j} \cdot r^{n-1} d r d \mathbf{l}=\sum_{j=0}^{\infty}(-1)^{j} \frac{(n-1+2 j) !}{2^{j} j !} \int_{O_{n}} \frac{1}{L^{n}}\left(\frac{Q}{L^{2}}\right)^{j} d \mathbf{l},
$$


after integrating with respect to $r$. Here $O_{n}$ is the surface of the unit $n$-sphere lying in the positive orthant. Denoting the angle between $\Delta$ and 1 by $\theta$ and the norm of $\Delta$ by $d$,

$$
\begin{aligned}
\cos \theta & =\Delta \mathrm{l}^{\prime} /\left(\Delta \Delta^{\prime}\right)^{1 / 2}=L /\left(\Delta \Delta^{\prime}\right)^{1 / 2}, \\
d & =\left(\Delta \Delta^{\prime}\right)^{1 / 2},
\end{aligned}
$$

the latter series is

$$
\frac{1}{d^{n}} \cdot \sum_{j=0}^{\infty}(-1)^{j} \frac{a_{j}}{d^{2 j}}
$$

where

$$
a_{j}=\frac{(n-1+2 j) !}{2^{j} j !} \int_{o_{n}}\left(\mathbf{M} \mathbf{l}^{\prime}\right)^{j}\left(\sec ^{n+2 j} \theta\right) d \mathbf{l}
$$

Since $a_{j}$ is a function only of $\mathbf{M}$ and of the orientation of $\Delta$ and is independent of the norm of $\Delta$, we conclude from (2.13) that (A) represents an asymptotic expansion of $R(\mathbf{a}, \mathbf{M})$ for positive $\Delta_{\alpha}$ and for large $\Sigma_{1}^{n} \Delta_{\alpha}^{2}$.

We conclude this section by remarking that the $\mu^{*}{ }_{i_{1}} \ldots i_{n}$ needed in the basic expansion (A) (the $\mu^{*} i_{1} \ldots i_{n}$ are defined in (2.4)) can be evaluated expeditiously by contraction (cf Kendall and Stuart [5], sec. 13.13, p. 319) and by exploitation of the well-known fact that the product of an even number of normal random variables with zero expectations can be expressed in terms of products of covariances between the variables. Specifically, let $Z_{1}, \ldots, Z_{2 j}$ have a joint normal distribution with zero expectation vector and arbitrary, not necessarily nonsingular, variancecovariance matrix. The property ${ }^{3}$ just stated in relation to the even order moments of a multinormal distribution is

$$
E\left[Z_{1} \ldots Z_{2 j}\right]=\Sigma^{\prime \prime} E\left[Z_{r_{1}} Z_{r_{2}}\right] \ldots E\left[Z_{r_{2 j-1}} Z_{r_{2 j}}\right]
$$

where $\left(r_{1}, r_{2}, \ldots, r_{2 j}\right)$ is a permutation of $(1,2, \ldots, 2 j)$ and $\Sigma^{\prime \prime}$ denotes summation over all possible ways of partitioning the set $\{1,2, \ldots, 2 j\}$ into $j$ subsets, each of size two, the number of such ways ${ }^{4}$ being $1.3 .5 \ldots \ldots(2 j-1)$. To evaluate $\left.E\left[X_{1}^{*}\right)^{i}{ }_{1} \ldots\left(X_{n}^{*}\right)_{n}{ }_{n}\right]$, where $i_{1}+\ldots+i_{n}=2 j$, by contraction from (2.14), set $^{5}$

$$
Z_{\alpha}=\left\{\begin{array}{lc}
X_{1}^{*}, \alpha=1,2, \ldots, i_{1} \\
X_{2}^{*}, \alpha=i_{1}+1, i_{1}+2, \ldots, i_{1}+i_{2} \\
\vdots & \vdots \\
X_{n}^{*} & \alpha=i_{1}+\ldots+i_{n-1}+1, i_{1}+\ldots+i_{n-1}+2, \ldots, 2 j
\end{array}\right.
$$

\section{An Important Special Case}

The problem of evaluating the probability that each of several standardized and equally correlated normal random variables shall not fall short of a specified value has been considered in some detail by various authors. Some applications of this problem, together with references relating to theoretical discussions are to be found in Ruben [11, 13]. (See also Bartholomew [1] for a further application.)

\footnotetext{
${ }^{3}$ It is of some interest that this property plays an important role in other theoretical applications in statistics, e.g., the mathematical theory of Brownian motion and thermal noise. (See Wang and Uhlenbeck [17], p. 332.)

${ }^{4}$ As an example, for $j=2$, we have the familiar result

$E\left[Z_{1} Z_{2} Z_{3} Z_{4}\right]=E\left[Z_{1} Z_{2}\right] E\left[Z_{3} Z_{4}\right]+E\left[Z_{1} Z_{3}\right] E\left[Z_{2} Z_{4}\right]+E\left[Z_{1} Z_{4}\right] E\left[Z_{2} Z_{3}\right]$

${ }^{5}$ As an example, to evaluate $E\left[\left(X_{1}^{*}\right)^{2} X_{2}^{*} X_{3}^{*}\right]$, set $Z_{1}=X_{1}^{*}, Z_{2}=X_{1}^{*}, Z_{3}=X_{2}^{*}, Z_{4}=X_{3}^{*}$, giving
} 
It may be useful to record here some of the main results available. Let $\mathbf{M}_{0}^{-1}$ denote an $n \times n$ matrix which has 1 for all its diagonal elements and $\rho$ for all its off-diagonal elements, and let $\mathbf{a}_{0}=(a, a, \ldots, a)$. Then in the notation of (1.1) and (1.2), for $\rho$ positive,

$$
F\left(\mathbf{a}_{0}, \mathbf{M}_{0}\right)=\int_{-\infty}^{\infty}\left[F\left(\frac{a-\rho^{1 / 2} x}{(1-\rho)^{1 / 2}}, 1\right)\right]^{n} f(x, 1) d x,
$$

$f(x, 1)$ and $F(x, 1)$ denoting the standardized normal density and distribution functions, respectively, evaluated at the point $x$. Formula (3.1) is a special case of a more general formula, due (independently) to Dunnett and Sobel [4], Das [3], and Stuart [15], in which $F(\mathbf{a}, \mathbf{M})$ is expressed as a univariate integral involving the standardized normal density and distribution functions when $\rho_{i j}$, the correlation between $X_{i}$ and $X_{j}$, is of the form $\rho_{i j}=\alpha_{i} \alpha_{j}(j \neq i)$, and a is arbitrary. Formula (3.1), specialized further by $a=0$, was proved (again independently) by Ruben [7] and Moran [6]. More recently, Steck ${ }^{6}$ and Owen [16] have shown that (3.1) may be extended to all $\rho$, positive or negative $(\rho>-1 /(n-1))$, the imaginary component of the right-hand integral being zero. Steck and Owen also provide valuable recursion relationships for $F\left(\mathbf{a}_{0}, \mathbf{M}_{0}\right)$.

Results of a rather more explicit character for $F\left(\mathbf{a}_{0}, \mathbf{M}_{0}\right)$ have been obtained by Ruben [11, 13]. In the first of these two papers, $R\left(\mathbf{a}_{0}, \mathbf{M}_{0}\right)$ was expressed as a convergent power series in $\mathbf{a}_{0} \mathbf{M a}_{0}^{\prime}$, the coefficients in the series being simple multiples of generalized centroids, or geometrical moments, of a regular $(n-1)$-dimensional regular spherical simplex with common dihedral angle arc $\cos (-\rho)$; these centroids are, in their turn, expressible (Ruben [8]) in terms of the contents of regular spherical simplices tabulated elsewhere (Ruben [7]). In the second paper, $R\left(\mathbf{a}_{0}, \mathbf{M}_{0}\right)$ was represented as an asymptotic expansion in negative powers of $\mathbf{a}_{0} \mathbf{M} \mathbf{a}_{0}^{\prime}$, the coefficients in the series being identical with the coefficients in the Taylor expansion of $\exp \left(-x^{2} / 2\right) K_{n-1}(x)$ at $x=0$, where $K_{n-1}(x)$ is the probability-mass, under a standardized $(n-1)$-dimensional spherical distribution, of a regular $(n-1)$-dimensional linear simplex with edges of length $x$ and with centroid at the center of the distribution. The purpose of this section is to verify that expansion (A) of the current paper, for the special case $F\left(\mathbf{a}_{0}, \mathbf{M}_{0}\right)$, agrees with that in [13], at any rate as far as the first three terms are concerned. (The two expansions in question are in fact completely identical.) At the same time this verification will serve to illustrate the use of the current expansion under more general conditions.

It is readily established that $\mathbf{M}$, the variance-covariance matrix of the conjugate distribution, has $\{1+(n-2) \rho\}\{1+(n-1) \rho\}^{-1}(1-\rho)^{-1}$ for its diagonal elements and $-\rho\{1+(n-1) \rho\}^{-1}(1-\rho)^{-1}$ for its off-diagonal elements (the common correlation in the conjugate distribution being consequently $\left.-\rho\{1+(n-2) \rho\}^{-1}\right)$. We find from (2.2) that the components of $\Delta$ are equal to (say) $\Delta$ defined by

$$
\Delta=a /\{1+(n-1) \rho\}
$$

whence $\Delta>0$, if, and only if, $a>0$. Assume, then, that $a>0$, that is, the cutoff point $\mathbf{a}_{0}$ lies on that part of the equiangular line $x_{1}=x_{2}=\ldots=x_{n}$ lying in the positive orthant. From (A.1) and (3.2),

$$
C_{j}=\left(\sum_{i_{1}}^{\sum} \ldots+i_{n=2 j} \mu_{i_{1}}^{*} \ldots i_{n}\right) \cdot\{1+(n-1) \rho\}^{2 j} / a^{2 j} \quad(j=0,1, \ldots) .
$$

Formulas (3.2) and (3.3) may now be used in formula (A) to evaluate $R\left(\mathbf{a}_{0}, \mathbf{M}_{0}\right)$ after the $\mu_{i_{1}}^{*} \ldots i_{n}$ have been determined. We proceed to evaluate $C_{1}$ and $C_{2}$ from (2.8) and (2.9).

Here $\mu_{i_{1}}^{*} \ldots i_{n}$ is unaffected by permutation of $i_{1}, \ldots i_{n}$, as is evident from the symmetry of the distribution. In particular, the moments occurring within any pair of braces in (2.8) and (2.9) are equal. Thus since

and

$$
\mu_{20}^{*} \ldots 0=\{1+(n-2) \rho\} /[\{1+(n-1) \rho\}(1-\rho)]
$$

$$
\mu_{110 \ldots 0}^{*}=-\rho /[\{1+(n-1) \rho\}(1-\rho)],
$$

${ }^{6}$ Since this paper was written, a further paper by Steck on orthant probabilities $(a=0)$ for the equicorrelated multivariate normal distribution has appeared (Biometrika 49, 433-445, 1962). 
we have from (2.8)

$$
\mathbf{C}_{1}=\left(n \mu_{20}^{*} \ldots 0+n(n-1) \mu_{110}^{*} \ldots 0\right) \cdot\{1+(n-1) \rho\}^{2} / a^{2}=n\{1+(n-1) \rho\}\{1+(n-3) \rho / 2\}(1-\rho)^{-1} / a^{2} .
$$

Again, from (2.9),

$\mathrm{C}_{2}=n \cdot \mu_{40}^{*} \ldots 0+n(n-1) \cdot \mu_{310}^{*} \ldots 0+\frac{1}{2} n(n-1) \cdot \mu_{220}^{*} \ldots 0+\frac{1}{2} n(n-1)(n-2) \cdot \mu_{2110}^{*} \ldots 0$

$$
+\frac{1}{24} n(n-1)(n-2)(n-3) \cdot \mu_{1110}^{*} \ldots 0
$$

where the moments occuring in $C_{2}$ are easily found (e.g., by contraction) to be given by

$$
\begin{aligned}
\mu^{*}{ }_{40} \ldots 0 & =3\{1+(n-2) \rho\}^{2} \gamma, \\
\mu *_{310} \ldots 0 & =-3 \rho\{1+(n-2) \rho\} \gamma, \\
\mu *_{220} \ldots 0 & =\left[\{1+(n-2) \rho\}^{2}+2 \rho^{2}\right] \gamma, \\
\mu^{*}{ }_{2110} \ldots 0 & =-\rho\{1+(n-4) \rho\} \gamma, \\
\mu *_{11110} \ldots 0 & =3 \rho^{2} \gamma,
\end{aligned}
$$

with $\gamma=\{1+(n-1) \rho\}^{-2}(1-\rho)^{-2}$. After some reduction

$$
C_{2}=n\{1+(n-1) \rho\}^{2}\left\{(5+n) / 2+\left(n^{2}+3 n-16\right) \rho / 2+\left(n^{3}+2 n^{2}-29 n+50\right) / 8\right\}(1-\rho)^{-2} / a^{4} .
$$

Higher order $C_{j}$ may be found similarly with correspondingly heavier algebra.

Our result is then

$$
\begin{array}{r}
F\left(\mathbf{a}_{0}, \mathbf{M}_{0}\right) \sim(2 \pi)^{-n / 2}\{1+(n-1) \rho\}^{-1 / 2}(1-\rho)^{-(n-1) / 2} e^{-n a^{2} /[2\{1+(n-1) \rho\}]} \\
. \frac{\{1+(n-1) \rho\}^{n}}{a^{n}}\left(C_{0}-C_{1}+C_{2}-\ldots\right),
\end{array}
$$

where $C_{1}$ and $C_{2}$ are given in (3.4) and (3.5) and $C_{0}=1$, the first term on the right of (3.6) representing $f\left(\mathbf{a}_{0}, \mathbf{M}_{0}\right)$, the probability density at the point $\mathbf{a}_{0}$. The first three terms in (3.6) agree with those of expansion (2.23) of [13] when the function $I_{n}(\mathrm{a}, \rho)$ in the latter paper is identified with the function $F\left(\mathbf{a}_{0}, \mathbf{M}_{0}\right)$ of the present paper. Also, as remarked earlier, the first two terms of the series (3.6) were derived by Savage [14] (example 3) as giving a lower bound to $F\left(\mathbf{a}_{0}, \mathbf{M}_{0}\right.$ ), while the first term of the series gives an upper bound. However, from property (i) in section 2 of this paper, all summands of the series in (3.6) with an odd number of terms yield upper bounds to $\boldsymbol{F}\left(\mathbf{a}_{0}, \mathbf{M}_{0}\right)$ and all summands with an even number of terms yield lower bounds.

\section{Scope of the Expansion and Possible Extension of Current Results}

It is clear from the form of $\Delta$ in (2.2) that expansion (A) for the multivariate normal integral will be particularly effective for large $a_{\alpha}, \alpha=1, \ldots, n$, i.e., when the distance between the cutoff point and the center of the distribution is large, and also under certain conditions of near degeneracy when $\mathbf{M}^{-1}$ is "almost" singular and the probability in the distribution of $\mathbf{X}$ is correspondingly highly concentrated around certain linear subspaces. For example, in the special case considered in section 3, where degeneracy occurs for $\rho=1$ and for $\rho=-1 /(n-1)$, we note from (3.2) that expansion (3.6) is most effective for high $a$ and for large negative $\rho$. (In this connection, compare the two sets of upper and lower bounds for $n=2, \mathbf{a}_{0}=(3,3)$ and $\rho= \pm 1 / 2$ given by Savage [14] in his example 1.)

In conclusion, we stress once more that expansion (A) is valid only for values of a such that $\mathbf{a M}>\mathbf{0}$. Geometrically, this means that the cutoff point $\mathbf{a}$ is restricted to the interior of a polyhedral half-cone bounded by the $n$ flats $\mathbf{x} \mathbf{M}=\mathbf{0}$ and with vertex at the center of the distribution. 
These flats are the regression planes (of $X_{1}$ on $X_{2}, \ldots, X_{n}$, of $X_{2}$ on $X_{1}, X_{3}, \ldots X_{n}$, etc.), and the statistical interpretation $\mathbf{a M}>\mathbf{0}$ is that the $n$ residuals of a relative to the $n$ regression planes are all positive. This is admittedly a rather severe restriction, and it would be desirable to have expansions, both asymptotic and otherwise, valid for at least positive values of the components of the cutoff point (i.e., for a lying in the positive orthant). It is hoped that the present paper may be a helpful step in this direction. (Special reference should here be made to S. S. Gupta's review article and extensive bibliography on the multivariate normal integral and related topics in the September 1963 issue of Annals of Mathematical Statistics. The reader is also referred to a recent paper by Curnow and Dunnett [2].) The restriction $\mathbf{a M}>\mathbf{0}$ is, however, not serious for the special case $n=2$, since it is known that the general bivariate normal integral with arbitrary cutoff point can be expressed in terms of the difference between two $F$-functions of the type discussed in section 3 (Ruben $[9,13]$ ).

That expansions of the sought for type exist (and the hunt for them therefore not chimerical) is readily established by methods virtually identical with those used in [11] and in section 3 of [13] for the equicorrelated case. In essence, this amounts to the following. The general multivariate normal integral is first identified with the probability-mass under a standardized spherical normal distribution of a polyhedral half-cone with angles between the bourding faces given by arc cos $\left(-\rho_{i j}\right)$ and with vertex $V$ at a distance $\zeta=\left(\mathbf{a M a}^{\prime}\right)^{1 / 2}$ from the center of the distribution. The intersection of the half-cone with a unit sphere centered at $V$ is a hyperspherical simplex with dihedral angles arc $\cos \left(-\rho_{i j}\right)$. Next, the required probability-mass in the half-cone is expressed as the product of $(2 \pi)^{-1 / 2} \exp \left(-\zeta^{2} / 2\right)$ and an infinite convergent power series in $\zeta$ with positive integral exponents, or, for ${ }^{7} \zeta>0$, as the product of $(2 \pi)^{-1 / 2} \exp \left(-\zeta^{2} / 2\right)$ and an asymptotic $(\zeta$ large), totally divergent power series in $\zeta$ with negative integral exponents, where the coefficients in the two series are simple multiples of certain integrals over the spherical simplex. The latter integrals are, in fact, of the form $\int \cos ^{j} \phi d$ in the one case and $\int \sec ^{n+2 j} \phi d l$ in the other case, where $\phi$ denotes the angle between a given fixed line and that line joining $V$ and a point on the surface of the sphere, while $d$ l is the surface-content of an infinitesimal element on the surface of the sphere. Unfortunately, however, these integrals appear to be intractable, and it is indeed clear that their evaluation will prove a formidable task, since even the special case $j=0$, in the first type of integrai, amounts to the determination of the content of the general hyperspherical simplex, i.e., to the solution of a long-standing and exceedingly difficult classical problem in $n$-dimensional geometry. (For references to the latter problem, which is equivalent to evaluating the probability in an orthant under a centered multivariate normal distribution, and for a discussion of the content of regular hyperspherical simplices, see [10] and [12].) Nevertheless, it may prove feasible to obtain the coefficients for certain correlation structures. Thus the coefficients have been determined, in [11] and [13], for the particularly simple correlation structure specified by the property "equality of correlations."

\section{References}

[1] D. J. Bartholomew, A test of homogeneity of means under restricted alternatives, J. Roy. Statist. Soc. Ser. B, 23, 239-281 (1961).

[2] R. N. Curnow and C. W. Dunnett, The numerical evaluation of certain multivariate normal integrals, Ann. of Math. Statist. 33, 571-579 (1962).

[3] S. C. Das, The numerical evaluation of a class of integrals, II, Proc. Cambridge Phil. Soc. 52, 442-448 (1956).

[4] C. W. Dunnett and M. Sobel, Approximations to the probability integral and certain percentage points of a multivariate analogue of Student's $t$-distribution, Biometrika 42, 258-260 (1955).

[5] M. G. Kendall and A. Stuart, The Advanced Theory of Statistics, Vol. 1 (Charles Griffin \& Co. Ltd., London, 1958).

[6] P. A. P. Moran, The numerical evaluation of a class of integrals, Proc. Cambridge Phil. Soc. 52, 230-233 (1956).

[7] Harold Ruben, On the moments of order statistics in normal samples. Biometrika 41, 200-227 (1954).

${ }^{7} \mathrm{By} \zeta>0$, we mean that the center of the distribution is exterior to the half-cone. 
[8] Harold Ruben, On the geometrical moments of skew-regular simplices in hyperspherical space, with some applications in geometry and mathematical statistics, Acta Mathematica, 103, 1-23 (1960).

[9] Harold Ruben, The probability content of regions under spherical normal distribution, II: The bivariate normal integral, Ann. Math. Statist. 32, 171-186 (1961).

[10] Harold Ruben, A power series expansion for a class of Schläfli functions. J. London Math. Soc. 36, 69-77 (1961).

[11] Harold Ruben, On the numerical evaluation of a class of multivariate normal integrals, Proc. Roy. Soc. of Edinburgh 65, 272-281 (1961).

[12] Harold Ruben, A multidimensional generalization of the inverse sine function, Quart. J. Math. 12, 257264 (1961).

[13] Harold Ruben, An asymptotic expansion for a class of multivariate normal integrals. J. Australian Math. Soc. 2, 253264 (1962).

[14] I. Richard Savage, Mill's ratio for multivariate normal distributions. J. Res. NBS 66B (Math. and Math. Phy.) No. 3, 93-96 (1962).

[15] A. Stuart, Equally correlated variables and the multinormal integral, J. Roy. Statist. Soc. Ser. B, 20, 373-378 (1958).

[16] G. P. Steck and D. B. Owen, A note on the equicorrelated multivariate normal distribution, Biometrika 49, 269-271 (1962).

[17] Ming Chen Wang and P. E. Uhlenbeck, On the theory of Brownian motion II, Rev. Modern Physics 17, 323-342 (1945).

(Paper 68B1-109) 